\title{
Erratum to: Production of Bioactive Volatiles by Different Burkholderia ambifaria Strains
}

\author{
Ulrike Groenhagen - Rita Baumgartner • Aurélien Bailly • \\ Amber Gardiner • Leo Eberl • Stefan Schulz • \\ Laure Weisskopf
}

Published online: 23 October 2013

(C) Springer Science+Business Media New York 2013

Erratum to: J Chem Ecol (2013)

DOI 10.1007/s10886-013-0315-y

The original version of this article unfortunately contained a mistake. In Table 2 on pages 901-902, the amounts of pure compounds used should read " $10 \mathrm{pg}, 10 \mathrm{ng}, 10 \mu \mathrm{g}$ " instead of " $1 \mathrm{ng}, 1 \mu \mathrm{g}, 1 \mathrm{mg}$ ". Below is the correct Table 2:

The online version of the original article can be found at http://dx.doi.org/ 10.1007/s10886-013-0315-y.

U. Groenhagen $\cdot$ S. Schulz $(\bowtie)$

Institute of Organic Chemistry, Technische Universität

Braunschweig, Hagenring 30, 38106 Braunschweig, Germany

e-mail: stefan.schulz@tu-bs.de

R. Baumgartner $\cdot$ A. Bailly $\cdot$ A. Gardiner $\cdot$ L. Eberl $\cdot$ L. Weisskopf Institute of Plant Biology, University of Zurich,

Zollikerstrasse 107, 8008 Zurich, Switzerland

L. Weisskopf $(\bowtie)$

Federal Research Station Agroscope Reckenholz-Tänikon,

Reckenholzstrasse 191, 8046 Zurich, Switzerland

e-mail: laure.weisskopf@agroscope.admin.ch 
Table 2 Effect of selected pure compounds on the growth of Arabidopsis thaliana (A. thal), on the growth of Rhizoctonia solani ( $R$. sol), Alternaria alternata (A. alt), Fusarium solani (F. sol) and the antibiotic resistance of Escherichia coli to Gentamicin (GM), Kanamycin (KM) and Ampicillin (AMP). The results are expressed in percentage of control treatment

\begin{tabular}{|c|c|c|c|c|c|c|c|c|}
\hline & Quantity & A. thal ${ }^{a}$ & R. sol ${ }^{b}$ & A. $a l t^{b}$ & F. sol ${ }^{b}$ & $\mathrm{GM}^{\mathrm{b}}$ & $\mathrm{KM}^{\mathrm{b}}$ & $\mathrm{AMP}^{\mathrm{b}}$ \\
\hline \multirow[t]{3}{*}{ Dimethyl disulfide (1) } & $10 \mathrm{pg}$ & 192 & 85 & 91 & 99 & 109 & 104 & 105 \\
\hline & $10 \mathrm{ng}$ & 227 & 101 & 94 & 98 & 111 & 101 & 79 \\
\hline & $10 \mu \mathrm{g}$ & 185 & 77 & 99 & 99 & 117 & 104 & 75 \\
\hline \multirow[t]{3}{*}{ 4-Methyl-2-pentanone (2) } & $10 \mathrm{pg}$ & 111 & 129 & 108 & 103 & 110 & 104 & 85 \\
\hline & $10 \mathrm{ng}$ & 23 & 124 & 108 & 99 & 104 & 99 & 98 \\
\hline & $10 \mu \mathrm{g}$ & 26 & 107 & 110 & 97 & 117 & 92 & 87 \\
\hline \multirow[t]{3}{*}{ 3-Hexanone (3) } & $10 \mathrm{pg}$ & 170 & 127 & 113 & 104 & 117 & 106 & 106 \\
\hline & $10 \mathrm{ng}$ & 206 & 135 & 102 & 100 & 114 & 87 & 99 \\
\hline & $10 \mu \mathrm{g}$ & 133 & 114 & 99 & 100 & 109 & 91 & 98 \\
\hline \multirow[t]{3}{*}{ 4-Heptanone (4) } & $10 \mathrm{pg}$ & 140 & 117 & 112 & 99 & 102 & 97 & 105 \\
\hline & $10 \mathrm{ng}$ & 135 & 125 & 111 & 102 & 114 & 82 & 108 \\
\hline & $10 \mu \mathrm{g}$ & 130 & 123 & 103 & 104 & 116 & 89 & 107 \\
\hline \multirow[t]{3}{*}{ 2,5-Dimethylpyrazine $(\mathbf{8})$} & $10 \mathrm{pg}$ & 148 & 103 & 102 & 101 & 103 & 94 & 98 \\
\hline & $10 \mathrm{ng}$ & 95 & 105 & 104 & 103 & 105 & 98 & 96 \\
\hline & $10 \mu \mathrm{g}$ & 79 & 101 & 105 & 102 & 107 & 103 & 108 \\
\hline \multirow[t]{3}{*}{ Dimethyl trisulfide (11) } & $10 \mathrm{pg}$ & 129 & 107 & 96 & 98 & 105 & 126 & 109 \\
\hline & $10 \mathrm{ng}$ & 143 & 89 & 100 & 97 & 100 & 110 & 112 \\
\hline & $10 \mu \mathrm{g}$ & 106 & 67 & 89 & 100 & 121 & 119 & 102 \\
\hline \multirow[t]{3}{*}{ 4-Octanone (12) } & $10 \mathrm{pg}$ & 100 & 97 & 95 & 100 & 110 & 113 & 107 \\
\hline & $10 \mathrm{ng}$ & 112 & 103 & 98 & 98 & 113 & 107 & 108 \\
\hline & $10 \mu \mathrm{g}$ & 51 & 72 & 92 & 95 & 111 & 106 & 108 \\
\hline \multirow[t]{3}{*}{ 4-Methylthio-2-butanone (13) } & $10 \mathrm{pg}$ & 48 & 96 & 85 & 99 & 114 & 102 & 82 \\
\hline & $10 \mathrm{ng}$ & 58 & 87 & 99 & 98 & 97 & 96 & 96 \\
\hline & $10 \mu \mathrm{g}$ & 59 & 84 & 90 & 93 & 113 & 97 & 82 \\
\hline \multirow[t]{3}{*}{ 1-Phenylethanol (16) } & $10 \mathrm{pg}$ & 104 & 105 & 107 & 100 & 101 & 92 & 97 \\
\hline & $10 \mathrm{ng}$ & 141 & 108 & 107 & 102 & 109 & 94 & 99 \\
\hline & $10 \mu \mathrm{g}$ & 128 & 114 & 96 & 102 & 115 & 99 & 104 \\
\hline \multirow[t]{3}{*}{$S$-Methyl methanethiosulphonate (17) } & $10 \mathrm{pg}$ & 102 & 89 & 96 & 100 & 94 & 95 & 108 \\
\hline & $10 \mathrm{ng}$ & 113 & 78 & 99 & 97 & 98 & 86 & 102 \\
\hline & $10 \mu \mathrm{g}$ & 91 & 73 & 100 & 104 & 94 & 109 & 103 \\
\hline \multirow[t]{3}{*}{ Acetophenone (18) } & $10 \mathrm{pg}$ & 234 & 87 & 92 & 100 & 88 & 108 & 110 \\
\hline & $10 \mathrm{ng}$ & 306 & 91 & 99 & 98 & 87 & 108 & 110 \\
\hline & $10 \mu \mathrm{g}$ & 241 & 78 & 100 & 103 & 104 & 97 & 104 \\
\hline \multirow[t]{3}{*}{ 1-(Methylthio)-3-pentanone (20) } & $10 \mathrm{pg}$ & 45 & 96 & 102 & 100 & 102 & 120 & 105 \\
\hline & $10 \mathrm{ng}$ & 53 & 91 & 98 & 99 & 112 & 109 & 102 \\
\hline & $10 \mu \mathrm{g}$ & 44 & 85 & 97 & 104 & 122 & 120 & 103 \\
\hline \multirow[t]{3}{*}{ 2-Nonanone (21) } & $10 \mathrm{pg}$ & 36 & 103 & 98 & 100 & 74 & 83 & 94 \\
\hline & $10 \mathrm{ng}$ & 91 & 90 & 92 & 99 & 76 & 86 & 89 \\
\hline & $10 \mu \mathrm{g}$ & 41 & 80 & 88 & 97 & 84 & 94 & 78 \\
\hline \multirow[t]{3}{*}{ Phenylpropan-1-one (24) } & $10 \mathrm{pg}$ & 47 & 89 & 102 & 98 & 118 & 109 & 102 \\
\hline & $10 \mathrm{ng}$ & 72 & 88 & 101 & 99 & 114 & 106 & 105 \\
\hline & $10 \mu \mathrm{g}$ & 117 & 80 & 100 & 102 & 108 & 114 & 105 \\
\hline \multirow[t]{3}{*}{ 1-Phenyl-1,2-propanedione (25) } & $10 \mathrm{pg}$ & 121 & 91 & 100 & 98 & 77 & 88 & 92 \\
\hline & $10 \mathrm{ng}$ & 71 & 98 & 91 & 96 & 78 & 79 & 92 \\
\hline & $10 \mu \mathrm{g}$ & 67 & 58 & 89 & 97 & 88 & 84 & 96 \\
\hline \multirow[t]{2}{*}{ 2-Undecanone (28) } & $10 \mathrm{pg}$ & 52 & 98 & 92 & 102 & 94 & 97 & 101 \\
\hline & $10 \mathrm{ng}$ & 53 & 60 & 89 & 100 & 98 & 88 & 105 \\
\hline
\end{tabular}


Table 2 (continued)

\begin{tabular}{|c|c|c|c|c|c|c|c|c|}
\hline & Quantity & A. thal ${ }^{a}$ & R. sol ${ }^{b}$ & A. $a l t^{b}$ & F. sol ${ }^{b}$ & $\mathrm{GM}^{\mathrm{b}}$ & $\mathrm{KM}^{\mathrm{b}}$ & $\mathrm{AMP}^{\mathrm{b}}$ \\
\hline & $10 \mu \mathrm{g}$ & 89 & 48 & 82 & 98 & 94 & 85 & 92 \\
\hline \multirow[t]{3}{*}{$o$-Aminoacetophenone (29) } & $10 \mathrm{pg}$ & 165 & 111 & 92 & 104 & 122 & 120 & 109 \\
\hline & $10 \mathrm{ng}$ & nd & 102 & 91 & 102 & 115 & 116 & 109 \\
\hline & $10 \mu \mathrm{g}$ & 175 & 98 & 104 & 100 & 124 & 121 & 99 \\
\hline \multirow[t]{3}{*}{ 2-Tridecanone (35) } & $10 \mathrm{pg}$ & 97 & 114 & 94 & 99 & 110 & 112 & 99 \\
\hline & $10 \mathrm{ng}$ & 64 & 97 & 92 & 100 & 107 & 107 & 92 \\
\hline & $10 \mu \mathrm{g}$ & 53 & 72 & 95 & 98 & 104 & 110 & 97 \\
\hline
\end{tabular}

Significant values are listed in bold, according to one-way ANOVA followed by Dunnett's post-hoc test $\left(p<0.05,{ }^{\mathrm{a}} n=20-25\right.$, $\left.{ }^{\mathrm{b}} n=3-4\right)$. nd: not determined 